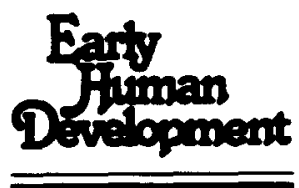

\title{
Doppler colour flow imaging of fetal intracerebral arteries relative to fetal behavioural states in normal pregnancy
}

\author{
Marja J. Noordam ${ }^{a}$, Franciska M.E. Hoekstra ${ }^{a}$, \\ Wim C.J. Hop ${ }^{b}$, Juriy W. Wladimiroff*a \\ a Department of Obstetrics and Gynaecology, 'Department of Biostatistics, \\ Academic Hospital Rotterdam-Dijkzigt, Erasmus University, Dr. Molewaterplein 40. \\ 3015 GD Rotterdam, The Netherlands
}

Received 7 February 1994; revision received 7 June 1994; accepted 13 June 1994

\begin{abstract}
In 14 normally developing term fetuses, the relationship between the blood flow velocity waveforms at cerebral arterial level (internal carotid artery, anterior, middle and posterior cerebral artery) and fetal behavioural states was studied using Doppler colour flow imaging. Behavioural state dependent changes in absolute flow velocities occurred in all vessels, except for the middle cerebral artery. These changes suggest preferential blood flow to the left heart resulting in increased flow to the cerebrum during fetal behavioural state $2 F$ (active sleep) when compared with fetal behavioural state IF (quiet sleep). The middle cerebral artery supplies the neocerebrum. This developing part of the cerebrum does not seem to take part in the regulation of fetal behaviour. In the internal carotid artery, an inverse relationship between peak systolic velocity and fetal heart rate could be established, which can be explained by a shorter rapid filling phase at raised fetal heart rate according to the Frank-Starling Law.
\end{abstract}

Keywords: Doppler colour flow imaging; Fetal behaviour; Fetal intracerebral arteries; Blood flow velocity

\section{Introduction}

Combined use of real-time and pulsed Doppler systems has now resulted in a large number of studies on fetal cerebral blood flow [4,18-21]. Fetal behavioural state de-

\footnotetext{
* Corresponding author.
} 
pendent changes are demonstrated in the descending aorta [3], foramen ovale [5], ductus venosus [8] and internal carotid artery [4]. Doppler colour flow imaging will allow accurate determination of direction of flow movement [18] and therefore angle dependent measurements of absolute flow velocities.

The objective of the present study was (i) to detect whether behavioural state dependent changes in absolute flow velocities in the intracerebral arteries are present in normal term fetuses, (ii) to establish if these changes are constant in all arteries of the circle of Willis and (iii) to detect a relationship between absolute flow velocity and fetal heart rate.

\section{Materials and methods}

A total of 14 women with singleton and uncomplicated pregnancies at 38-39 weeks of gestation consented to participate in the study. The gestational age had been calculated from the last menstrual period and confirmed by ultrasonic measurements of fetal crown-rump length or biparietal diameter. All participants were non-smokers and no medication was used. Fetal abdominal circumference was between the 10th and 90th percentile of the reference chart [1] and birth weight between the 10th and 90th percentile for gestational age according to Kloosterman's tables [10], corrected for maternal parity and fetal sex. There were no structural anomalies. The protocol of the study had been approved by the Hospital Ethics Committee.

A Toshiba SSA $270 \mathrm{~A}$ with a combined curved-linear two-dimensional real-time and pulsed Doppler 3.75-MHz probe was used. The Doppler recordings were performed by one examiner (MJN). In each woman, an attempt was made to document Doppler colour flow patterns in the fetal middle cerebral artery (MCA), internal carotid artery (ICA), anterior cerebral artery (ACA) and posterior cerebral artery (PCA). The technique of colour flow imaging to identify the intracranial vasculature has been described previously [18]. A transverse scan through the lower part of the fetal cerebrum shows a heart-shaped cross-section of the brain stem with the anterior lobes representing the pedunculi cerebri [21]. Anterior to this heart-shaped structure and on either side of the mid-line, the anterior cerebral arteries can be seen. The middle cerebral artery can be identified as a major branch of the circle of Willis running anterolaterally towards the lateral edge of the orbit. The internal carotid artery is visualized at its bifurcation into the middle and anterior branches. The postcrior cerebral arteries can be detected laterally to the cerebral peduncles.

Sample volume length ranged between 0.1 and $0.3 \mathrm{~cm}$. The correct position of the pulsed Doppler gate was ensured by two-dimensional ultrasound. Doppler tracings in the intracranial arteries were accepted when the angle between the Doppler cursor and the direction of flow was $10^{\circ}$ or less.

All Doppler studies were carried-out $2 \mathrm{~h}$ after breakfast or lunch, with the woman in a semirecumbent position and during periods of fetal apnoea. Minimal transducer pressure was applied to the maternal abdomen, as fetal head compression is associated with alterations in the fetal intracranial arterial flow velocity waveform [19].

Maximum flow velocity waveforms were recorded during fetal behavioural states 
(FBS) $1 \mathrm{~F}$ and $2 \mathrm{~F}$ according to the classification of Nijhuis et al. [12]. These behavioural states are defined as follows: (1) State $1 \mathrm{~F}$ (quiet sleep) - quiescence, which can be regularly interrupted by brief gross body movements, which mostly are startles; absent eye movements and a stable heart rate pattern with a narrow oscillation bandwidth. Isolated accelerations do occur, but these are strictly related to movements. (2) State $2 \mathrm{~F}$ (active sleep) - frequent and periodic gross body movements that are mainly stretches and retroflexions and movements of extremities; eye movements almost continually present; a heart rate pattern with a wider oscillation bandwidth than in state $1 \mathrm{~F}$ and frequent accelerations during movements.

In order to establish fetal behavioural states, the following parameters were simultaneously recorded: (i) The fetal heart rate (FHR), which was obtained from a Doppler ultrasound cardiotocograph (Hewlett Packard 8040A, carrier frequency $1 \mathrm{MHz}$ ); (ii) Fetal eye movements, which were observed by ultrasonic visualisation of the fetal eye lens in a transverse scanning plane through the orbits using the twodimensional real-time scanner. Immediately after establishing the presence or absence of eye movements, the transducer was moved in a sagittal scanning plane of the fetal trunk to confirm the presence of body movements.

Flow velocity recordings were only performed when a clear fetal behavioural state had been identified and when this state had been present over a period of at least $3 \mathrm{~min}$. Blood flow velocity waveforms were recorded on videotape, from which hard copies were made. A microcomputer (Olivetti M24), linked to a graphics tablet was used for analysis of the Doppler recordings. Waveform analysis was performed by an independent examiner (FMEH). Peak systolic (PSV, $\mathrm{cm} / \mathrm{s}$ ), end-diastolic (EDV, $\mathrm{cm} / \mathrm{s})$, and time-averaged $(\mathrm{AV}, \mathrm{cm} / \mathrm{s})$ velocities were determined in all four intracerebral vessels. Period time (msec) was established from the time interval between peak systoles from two consecutive cardiac cycles. From this, the fetal heart rate (FHR) was calculated. An average of at least three consecutive flow velocity waveforms of optimal quality was used to establish each value.

The relationship between the various Doppler parameters on the one hand and FBS ( $1 \mathrm{~F}$ and $2 \mathrm{~F}$ ) and fetal heart rate on the other hand were evaluated using repeated measurement analysis of variance [17]. For each Doppler parameter, the mean slope and intercept of individual regression lines for both behavioural states were determined using an iterative search for optimal values [6]. After verifying that the slopes thus derived did not significantly differ between FBS $1 F$ and $2 F$, the difference in intercepts of the two regression lines which were taken to run parallel, was determined to estimate the FHR-adjusted difference in mean outcome between both behavioural states. This adjustment was necessary because in FBS $2 F$, the fetal heart rate was generally higher compared to FBS $1 \mathrm{~F}$. Statistical significance was tested at the level of 0.01 (two-sided).

\section{Results}

A total of 508 tcchnically acceptablc blood flow vclocity recordings from the intracerebral arteries were analysed. The success rate in obtaining good quality Doppler flow velocity waveforms for both fetal behavioural states was $100 \%$ for the 
MCA, $96.4 \%$ for the ICA, $92.8 \%$ for the ACA and $82.1 \%$ for the PCA. An even distribution of recordings was available for analysis. In the $\mathrm{MCA}$, the median number of recordings was five (range 4-7) in FBS $1 \mathrm{~F}$ and five (range 4-10) in FBS $2 \mathrm{~F}$; in the ICA, these numbers were four (range 2-7) and five (range 0-5); in the ACA, five (range 0-6) and five (range 0-9) and in the PCA, five (range 0-7) and five (range $0-6$ ).

Fig. 1 is an example of the applied method of analysis. The measurements of the peak systolic velocity in the ICA obtained in FBS $1 F$ and $2 \mathrm{~F}$ are given, as well as the regression lines corresponding to the two fetal behavioural states. As is also demonstrated in Table 1, a statistically significant mean difference in peak systolic velocity existed in the ICA between FBS $1 F$ and 2 F $(P<0.01)$. ICA PSV demonstrated a statistically significant reduction $(P<0.01)$ with increasing fetal heart rate. Table 1 also presents the mean difference between FBS $1 F$ and $2 F$ for each of the blood flow velocity parameters studied, as well as the slope for the relation between each blood flow velocity parameter and fetal heart rate.

Statistical significance was reached for the mean difference between FBS IF and $2 \mathrm{~F}$ for all ICA parameters $(P<0.01)$, all ACA parameters $(P<0.01)$, PCA PSV $(P<0.001)$, and PCA AV $(P=0.001)$. No significant difference was found for MCA velocity parameters.

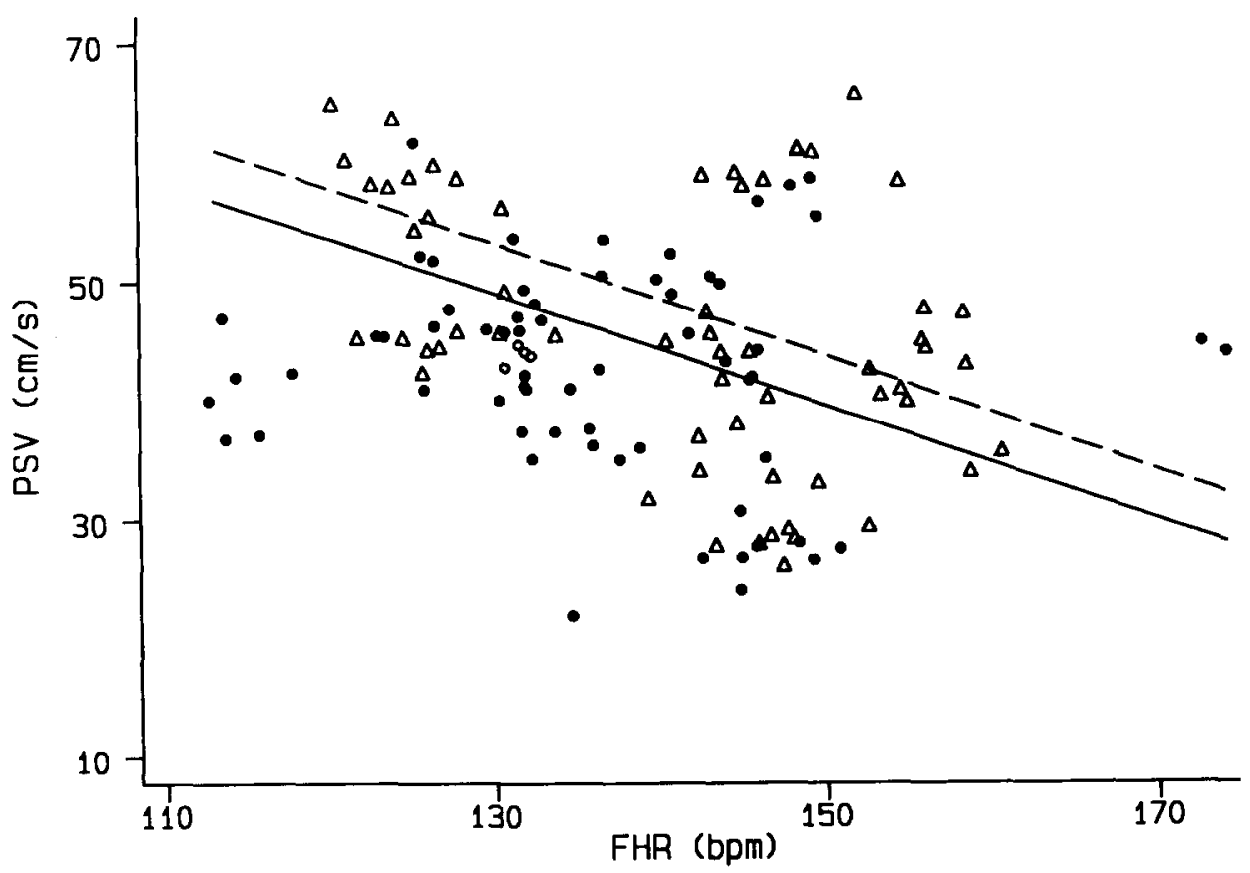

Fig. 1. ICA PSV measurements obtained in FBS $1 F(0)$ and in FBS $2 F(\Delta)$. Solid and interrupted lines denote regression lines corresponding to the two respective behavioural states. 
Table 1

Mean difference between FBS 2F and FBS IF for all intracerebral arterial flow velocities studied, as well as the slope for the relation between each blood fow velocity parameter and fetal heart rate

\begin{tabular}{lllll}
\hline Parameter & $\begin{array}{l}\text { Mean } \\
\text { difference } \\
2 \mathrm{~F}-1 \mathrm{~F} \\
(\mathrm{~cm} / \mathrm{s})\end{array}$ & $\begin{array}{l}\text { Significance of } \\
\text { difference } \\
(P \text {-value })\end{array}$ & $\begin{array}{l}\text { Slope } \\
([\mathrm{cm} / \mathrm{s}] / \\
{[\text { beats/min] })}\end{array}$ & $\begin{array}{l}\text { Significance } \\
\text { of slope } \\
(P \text {-value })\end{array}$ \\
\hline Middle cerebral artery & & & & \\
PSV & -0.96 & 0.38 & -0.32 & 0.06 \\
AV & 0.76 & 0.25 & -0.22 & 0.11 \\
EDV & 1.20 & 0.02 & -0.13 & 0.23 \\
Internal carotid artery & & & & \\
PSV & 3.80 & $<0.01$ & -0.47 & $<0.01$ \\
AV & 2.00 & $<0.01$ & -0.16 & 0.15 \\
EDV & 0.98 & $<0.01$ & 0.01 & 0.84 \\
Anterior cerebral artery & & & & \\
PSV & 4.48 & $<0.01$ & 0.21 & 0.38 \\
AV & 3.00 & $<0.01$ & 0.20 & 0.15 \\
EDV & 1.80 & $<0.01$ & 0.14 & 0.04 \\
Posterior cerebral artery & & & & \\
PSV & 4.50 & $<0.001$ & 0.03 & 0.72 \\
AV & 1.70 & 0.001 & 0.06 & 0.24 \\
EDV & 0.29 & 0.36 & 0.05 & 0.11 \\
\hline
\end{tabular}

PSV, peak systolic velocity; AV, time averaged velocity; EDV, end-diastolic velocity.

\section{Discussion}

The entire brain is supplied by two pairs of arterial trunks, the internal carotid arteries and the vertebral arteries. Lateral to the optic chiasm, the internal carotid artery divides into a smaller anterior cerebral artery and a larger middle cerebral artery. The middle cerebral artery is regarded as the direct continuation of the internal carotid artery. The vertebral artery arises from the first part of the subclavian artery and courses along the anterolateral surface of the medulla. Both vertebral arteries unite at the caudal border of the pons to form the basilar artery. The posterior cerebral arteries are formed by the bifurcation of the basilar artery at the rostral border of the pons. The circle of Willis is formed by anastomotic branches of the internal carotid artery and the most rostral branches of the basilar artery (i.e. the posterior cerebral artery).

Recording of blood flow velocity waveforms of different fetal intracerebral arteries has been reported with conventional real time ultrasound and pulsed Doppler [20]. Using Doppler colour flow imaging however, better visualization of the circle of Willis is achieved [18]. Whilst with conventional real time ultrasound only arterial vessel wall pulsations can be visualized, with Doppler colour flow imaging, proper identification of blood flow direction in a particular vessel is obtained, allowing angle dependent measurements of absolute flow velocities.

In the last weeks of pregnancy, identifiable fetal behavioural states have become 
apparent [12]. Behavioural state has been shown to influence cerebral blood flow in animals [14], human neonates [11] and fetuses [4], i.e. an increase during rapid eye movement sleep (active sleep, FBS 2F) when compared to non-rapid eye movement (quiet sleep, FBS 1F).

The internal carotid artery is one of the most important vessels supplying the fetal brain. All three velocities i.e. the peak systolic, averaged and end-diastolic velocity were significantly increased in FBS $2 \mathrm{~F}$ compared to FBS $1 \mathrm{~F}$. The increased enddiastolic velocity suggests a reduced cerebral vascular resistance during FBS $2 F$, while the increased peak systolic and averaged velocity could be explained by an increased contraction force of the heart or a redistribution of blood flow during FBS $2 \mathrm{~F}$ in favour of the left heart. As suggested in earlier reports [8], a redistribution of volume flow at the level of the umbilical sinus should be considered with increased flow in the ductus venosus during FBS 2F compared with FBS 1F. This is consistent with other studies suggesting an FBS $2 F$ related rise in volume flow at foramen ovale [5] and mitral valve level [16]. Increased volume flow through the left heart would be necessary to ensure raised cerebral blood flow during FBS $2 F$ which has been demonstrated in animal studies $[9,15]$ and is also suggested from data on reduced vascular resistance at cerebral level in the human fetus [4].

In the middle cerebral artery, no significant behavioural state related change in flow velocities was found. This could be attributed to the fact that the middle cerebral artery supplies the neocerebrum, i.e. the developing cerebral hemispheres or midbrain. Although in term fetuses, the complex pattern of sulci and gyri is already developed, myelinisation is still incomplete and the number of synapses is still increasing [13]. It is therefore not surprising that no significant difference between FBS $1 \mathrm{~F}$ and $2 \mathrm{~F}$ is demonstrated in this immature part of the cerebrum. This is in agreement with the conclusion of Chugani et al. [2] which says that according to the PET scan, increased glucose metabolic activity in the parietal, temporal and occipital cortices does not occur before the age of 3 months. Since middle cerebral artery flow velocity waveforms are not subject to fluctuation with fetal behavioural state as are the other intracerebral waveforms, it may be the most useful vessel to study for clinical purposes.

All three parameters i.e. peak systolic, averaged and end-diastolic velocity in the anterior cerebral artery were significantly increased in FBS $2 F$, which together with the presence of rapid-eye movements suggests raised electro-cortical activity in the frontal lobes. The posterior cerebral artery is responsible for the oxygen supply to the medulla, pons and part of the cerebellum. The significant increase in peak systolic and averaged velocity in the posterior cerebral artery during FBS $2 F$ could also be explained by the earlier mentioned increased contraction force of the heart or redistribution in blood flow at cardiac level during FBS 2F. The end-diastolic velocity is not significantly different between FBS $2 F$ and $1 F$, indicating that peripheral resistance has not changed. These data however, are at variance with the reported role of the pons in the regulation of sleep states in the fetal lamb $[9,15]$.

Van Eyck et al. $[3,4]$ established a decrease in PI in the umbilical artery and fetal descending aorta with rising fetal heart rate. This inverse relationship is mainly determined by the definition of Gosling and King [7] for PI calculations, i.e. at a 
lower FHR a more gradual end-diastolic slow-down of the blood flow velocity takes place.

The inverse relationship between peak systolic velocity in the internal carotid artery and FHR could be explained by the shorter ventricular filling phase at raised FHR and vice versa as dictated by the Frank-Starling Law. In the other intracerebral arteries, this effect could not be established.

It can be concluded that Doppler colour flow imaging allows absolute measurements of fetal cerebral flow velocities. Fetal behavioural state dependant changes occur at cerebral arterial level which are in agreement with changes observed at cardiac and venous inflow level.

\section{References}

[1] Campbell, S. and Wilkin, D. (1975): Ultrasonic measurement of fetal abdomen circumference in estimation of fetal weight. Br. J. Obstet. Gynaecol., 82, 689-697.

[2] Chugani, H.T., Phelps, M.E. and Mazziotta, J.C. (1987): Positron emission tomography study of human brain functional development. Ann. Neurol., 22, 487-497.

[3] Eyck, J. van, Wladimiroff, J.W., Noordam, M.J., Tonge, H.M. and Prechtl, H.F.R. (1985): The blood flow velocity waveform in the fetal descending aorta; its relationship to fetal behavioural states in normal pregnancy at 37-38 weeks of gestation. Early Hum. Dev., 12, 137-143.

[4] Eyck, J. van, Wladimiroff, J.W., Wijngaard, J.A.G.W. van den, Noordam, M.J. and Prechtl, H.F.R. (1987): The blood flow velocity waveform in the fetal internal carotid and umbilical artery; its relationship to fetal behavioural states in normal pregnancy at 37-38 weeks of gestation. $\mathrm{Br}$. $\mathrm{J}$. Obstet. Gynaecol., 94, 736-741.

[5] Eyck, J. van, Stewart, P.A. and Wladimiroff, J.W. (1990): Human fetal foramen ovale flow waveforms relative to behavioural states in normal term pregnancy. Am. J. Obstet. Gynccol., 163, 1239-1242.

[6] Feldman, H.A. (1988): Families of lines: random effects in linear regression analysis. J. Appl. Physiol., 64, 1721-1732.

[7] Gosling, R.G. and King, D.H. (1975): Ultrasonic angiology. In: Arteries and Veins, pp. 61-98. Editors: A.W. Marcus and L. Adamson. Churchill Livingstone, Edinburgh.

[8] Huisman, T.W.A., Brezinka, Ch., Stewart, P.A., Stijnen, Th. and Wladimiroff, J.W. (1994): Human fetal ductus venosus flow velocity waveforms relative to fetal behavioural states in normal term pregnancy. Br. J. Obstet. Gynaecol., 101, 220-224.

[9] Jensen, A., Bamford, O.S., Dawes, G.S., Hofmeyer, G. and Parkes, M.J. (1985): Changes in organ blood flow between high and low voltage electrocortical activity and during isocapnic hypoxia in intact and brainstem transected fetal lambs. In: The Physiological Development of the Fetus and Newborn, pp. 605-610. Editors: C.T. Jones and P.W. Nathanielsz. Academic Press, London.

[10] Kloosterman, G.J. (1970): On intrauterine growth. Int. J. Gynaecol. Obstet., 8, 895-912.

[11] Mukhtar, A.I., Cowan, F.M. and Stothers, J.K. (1982): Cranial blood flow and blood pressure changes during sleep in the human neonate. Early Hum. Dev., 6, 59-64.

[12] Nijhuis, J.G., Prechtl, H.F.R., Martin, C.B., Jr. and Bots, R.S.G.M. (1982): Are there behavioural states in the human fetus? Early Hum. Dev., 6, 177-195.

[13] Pomeroy, S.L. and Volpe, J.J. (1992): Development of the nervous system. In: Fetal and Neonatal Physiology, Vol. I1, pp. 1490-1509. Editors: R.A. Polin and W.W. Fox. Saunders, Philadelphia, PA.

[14] Reivich, M., Isaacs, G., Evarts, E. and Kety, S. (1968): The effect of slow wave sleep and REM sleep on regional cerebral blood flow in cats. J. Neurochem., 15, 301-306.

[15] Richardson, B.S., Patrick, J.E. and Abduljabbar, H. (1985): Cerebral oxidative metabolism in the fetal lamb: relationship to electrocortical state. Am. J. Obstet. Gynecol., 153, 426-431.

[16] Rizzo, G., Arduini, D., Valensise, H. and Romanini, C. (1990): Effects of behavioural states on cardiac output in the healthy human fetus at 36-38 weeks of gestation. Early Hum. Dev., 23, 109-115. 
[17] Schluchter, M.D. (1990): Unbalanced repeated measure models with structured covariance matrices. In: BMDP Statistical Software Manual, Vol. 2, pp. 1207-1244. Editor: W.J. Dixon. University California Press, Berkeley, CA.

[18] Vyas, S., Nicolaides, K., Bower, S. and Campbell, S. (1990a): Middle cerebral artery flow velocity waveforms in fetal hypoxaemia. Br. J. Obstet. Gynaecol., 97, 797-800.

[19] Vyas, S. Campbell, S. and Bower, K.H. (1990b): Maternal abdominal pressure alters fetal cerebral blood flow. Br. J. Obstet. Gynaecol., 97, 740-742.

[20] Wijngaard, J.A.G.W. van den, Groenenberg, I.A.L., Wladimiroff, J.W. and Hop, W.C.J. (1989): Cerebral Doppler ultrasound of the human fetus. Br. J. Obstet. Gynaecol., 96, 845-849.

[21] Wladimiroff, J.W., Tonge, H.M. and Stewart, P.A. (1986): Doppler ultrasound assessment of cerebral blood flow in the human fetus. Br. J. Obstet. Gynaecol., 93, 471-475. 